\title{
Some Higher Order Integral Identities With Application to Bounding Techniques*
}

\author{
J. H. Bramble ${ }^{1}$ and B. E. Hubbard ${ }^{2}$
}

(July 17, 1961)

\begin{abstract}
Let $R$ be a simply connected region in $E_{N}$ with smooth bounding surface $S$. For a sufficiently differentiable set of functions we derive a class of quadratic integral identities relating surface integrals of derivatives to integrals over $R$. These identities are a generalization of a first order identity given by L. Hörmander (Compt. Rend. Douzième Congr. des Mathématiciens Scandinaves Tenu à Lund, 1953, pp. 105-115) and L. E. Payne and H. F. Weinberger (Pacific J. Math. (1958) pp. 551-573). As an example of an application of these identities we consider a solution $u$ of the boundary value problem $\Delta u-p u=F$ in $R$ and $u=f$ on $S$. Here $\Delta$ denotes the Laplace operator and $0 \leq p(x)$. We obtain pointwise a priori bounds for the derivatives of $u$ in $R$ in terms of a quadratic functional of an arbitrary function. Hence the Rayleigh-Ritz procedure can be used to make the error arbitrarily small.
\end{abstract}

\section{Introduction}

In a recent paper $[6]^{3}$ L. E. Payne and H. F. Weinberger give a method for obtaining bounds for solutions of second order elliptic boundary value problems. In that paper they give a generalization (see eq 2.4) of an integral identity of Rellich [7] which was essential to their method. This generalization, which was first obtained and applied to hyperbolic operators by L. Hörmander [4], displays the highest derivatives in the form of a second order operator $L u \equiv\left(a^{i j} u,{ }_{j}\right)_{i}$. This fact makes the identity useful in treating boundary problems for the corresponding differential equation $L u=F$.

Use was also made of this identity by Hubbard [5] to obtain bounds for membrane eigenvalues by finite difference methods.

In section 2 we give a further generalization of Hörmander's result, which involves higher derivatives on the boundary and where the highest order terms enter as derivatives of $L u$. Section 3 gives an application of these higher order identities in obtaining pointwise bounds for the derivatives of a function $u$. The particular problem treated assumes a knowledge of $\Delta u-p u$ in a region $R$ and $u$ on the boundary $C$ where $\Delta$ is Laplace operator and $p>0$ in $R+C$. Because of the important physical application of this'equation (see e.g.,'Bergman and Schiffer [1]), this problem was chosen as an example to illustrate a use of the higher order identity. More general equations could be treated with only technical modifications. Other methods for obtaining explicit pointwise bounds for derivatives in such problems have been given by J. B. Diaz [3] and by Payne and Weinberger [6]. In both cases the method given involves differentiation of the funda-

\footnotetext{
*This research was supported in part by the Naval Ordnance Laboratory through the Fundamental Research Program and in part by the United States Air Force through the Air Force Office of Scientific Research of the Air Research and Development Command under Contract No. AF 49(638)-228.

1 University of Maryland; Consultant, NBS.

2 U.S. Naval Ordnance Laboratory. Present address, University of Maryland, College Park, Md.

${ }^{3}$ Figures in brackets indicate the literature references at the end of this paper.
}

mental solution in Green's third identity, thus introducing more singular behavior of the bounds as the boundary is approached. In our paper the bounds for derivatives of all orders have the same behavior near the boundary, provided the boundary data is sufficiently differentiable.

Extensions of the fundamental identity (2.4) can be derived for higher order operators by the same technique used here. One such, involving the biharmonic operator $\Delta^{2}$, is applied in a forthcoming paper of J. H. Bramble and L. E. Payne [2] to obtain explicit pointwise bounds in the biharmonic Dirichlet problem.

As has been previously pointed out in various places (see e.g. [6]) bounds of the type obtained here can be used in conjunction with a Rayleigh-Ritz technique to approximate the unknown solution (or derivative) arbitrarily closely.

\section{A Class of Quadratic Integral Identities}

Let

$$
L(u)=\left(a^{i j} u,{ }_{j}\right)_{\mid i},
$$

be defined on a region $R$ in $E_{n}$ with boundary $C$. The symbol $i i$ indicates covariant differentiation which coincides with, ${ }_{i}$ (partial differentiation with respect to $x^{i}$ ) in Cartesian coordinates. A repeated index indicates summation from 1 to $N$. The symmetric tensor $a^{i j}(x)$ is assumed to possess piecewise continuous derivatives of order $M+1$. In addition, let the eigenvalues of the matrix $a^{i j}$ be bounded away from zero and infinity in $R$. Hence there exists a positive constant, $a$, such that for all real nonzero $\left(\xi_{1}, \ldots, \xi_{n}\right)$ and all $x$ in $R$,

$$
a^{-1} \sum_{i=1}^{N} \xi_{i}^{2} \leq a^{i j} \xi_{i} \xi_{j} \leq a \sum_{i=1}^{N} \xi_{i}^{2}
$$

Under these conditions $L$ is said to be uniformly elliptic. 
Let $f^{i}(x), A_{1}^{i j}(x), \ldots, A_{m}^{i j}(x)$ be symmetric tensors of the second order with piecewise continuous first derivatives in $R$. L. E. Payne and H. F. Weinberger have developed the following first order quadratic identity [6] and used it to obtain pointwise bounds in certain boundary problems. It is clear that

$$
\begin{aligned}
\left(f^{m} a^{i j} u_{\mid i} u_{\mid j}\right)_{\mid m}= & \left(f^{m} a^{i j}\right)_{\mid m} u_{\mid i} u_{\mid j}+2 f^{m} a^{i j} u_{\mid i} u_{\mid m j} \\
= & \left(f^{m} a^{i j}\right)_{\mid m} u_{\mid i} u_{\mid j}+2\left(f^{m} a^{i j} u_{\mid i} u_{\mid m}\right)_{\mid j} \\
& -2 f^{m} a^{i j} u_{\mid i} u_{\mid m}-2 f^{m} u_{\mid m} L(u) .
\end{aligned}
$$

An application of the divergence theorem yields

$$
\begin{array}{r}
\oint_{C}\left\{f^{m} a^{i j}-2 f^{j} a^{i m}\right\} u_{\mid i} u_{\mid j} \nu_{m} d s=-2 \int_{R} f^{m} u_{\mid m} L(u) d v \\
+\int_{R}\left\{\left(f^{m} a^{i j}\right)_{\mid m}-2 f_{\mid m}^{j} a^{i m}\right\} u_{\mid i} u_{\mid j} d v .
\end{array}
$$

In an analogous manner we can develop a homogeneous quadratic integral identity involving second derivatives of $u$ on the boundary $C$. Now

$$
\begin{aligned}
\left(f^{m} A^{i j} a^{k l} u_{\mid i k} u_{\mid \jmath l}\right)_{\mid m} & \\
= & \left(f^{m} A^{i j} a^{k l}\right)_{\mid m} u_{\mid i k} u_{\mid j l}+2 f^{m} A^{i j} a^{k l} u_{\mid i k} u_{\mid j m l} \\
= & +2\left(f^{m} A^{i j} a^{k l} u_{\mid i k} u_{\mid j m}\right)_{\mid l}-2 f^{m} A^{i j} u_{\mid j m}(L u)_{\mid \imath} \\
+ & \left\{\left(f^{m} A^{i j} a^{k l}\right)_{\mid m}-2\left(f^{l} A^{i j} a^{k m}\right)_{\mid m}\right\} u_{\mid i k} u_{\mid j l} \\
& +2 f^{m} A^{i j}\left(a_{\mid k}^{k l} u_{\mid l}\right)_{\mid i} u_{\mid \jmath m} .
\end{aligned}
$$

After applying the divergence theorem we have

$$
\begin{aligned}
\oint_{C} A^{k l} & \left\{f^{m} a^{i j}-2 f^{j} a^{i m}\right\} u_{\mid i k} u_{\mid j l} \nu_{m} d s \\
= & -2 \int_{R} f^{m} A^{i j} u_{\mid j m}(L u)_{\mid i} d v+2 \int_{R} f^{m} A^{i j}\left(a_{\mid l}^{k l} u_{\mid k}\right)_{\mid i} u_{\mid j m} d v \\
& +\int_{R}\left\{\left(f^{m} A^{i j} a^{k l}\right)_{\mid m}-2\left(f^{l} A^{i j} a^{k m}\right)_{\mid m}\right\} u_{\mid i k} u_{\mid j l} d v .
\end{aligned}
$$

The corresponding identity which involves covariant derivatives of order $M+1$ on the boundary is

$$
\begin{aligned}
& \oint_{C} A_{1}^{i_{1} j_{1}} \ldots A_{M}^{i_{M j} j_{M}}\left\{f^{m} a^{k l}-2 f^{l} a^{k m}\right\} u_{\mid k i_{1} \ldots i M} u_{i l j_{1} \ldots j_{M} \nu_{m}} d S \\
& =-2 \int_{R} f^{m} A_{1}^{i_{1} j_{1}} \ldots A_{M}^{i_{M j_{M}}}(L u)_{\mid i_{1} \ldots i_{M}} u_{\mid j_{1}} \ldots j_{M} m d v \\
& +\int_{R}\left\{\left(f^{m} A_{1}^{i_{1} j_{1}} \ldots A_{M}^{i_{M j} j_{M}} a^{k l}\right)_{m}\right. \\
& \left.-2\left(f^{l} A_{1}^{i_{1} j_{1}} \ldots A_{M}^{i_{M j} j_{M}} a^{k m}\right)_{\mid m}\right\} u_{\mid i_{1} \ldots i_{M} k} u_{\mid j_{1} \ldots j_{M} l} d v \\
& \left.+2 \int_{R} f^{m} A_{1}^{i_{1} j_{1}} \ldots A_{M}^{i_{M j} j_{M}}\left(a_{\mid k}^{k l} u_{! l}\right)\right)_{i_{1}} \ldots i_{M} u_{\mid j_{1}} \ldots j_{M} m d v .
\end{aligned}
$$

Since the integrands are in each case tensor invariants, we may perform each integration in the most advantageous coordina te system. Since boundary conditions usually are given in terms of normal and tangential derivatives we shall display the derivatives appearing in the boundary integrals in such a form. Assume $C$ to be a surface possessing $M+1$ continuous derivatives as given in the parametric form

$$
\chi^{i}=G^{i}\left(y^{1}, \ldots, y^{N-1}\right), \quad i=1, \ldots, N .
$$

The unit normal $X^{i}(y)$ satisfies the conditions

$$
\begin{gathered}
G^{i}{ }_{\alpha} X^{i}=0, \quad \alpha=1, \ldots, N-1, \\
X^{i} X^{i}=1
\end{gathered}
$$

and the orientation of $X^{i}$ is taken inward. In what follows Greek indices will always range over $1, \ldots$, $N-1$, whereas Latin indices will refer to $1, \ldots, N$.

We introduce geodesic normal coordinates $\left(y^{1}, \ldots\right.$, $\left.y^{N}\right)$ in a strip immediately adjacent to the bounding surface. The transformation is given by

$\chi^{i}=G^{i}\left(y^{1}, \ldots, y^{N-1}\right)+y^{N} X^{i}\left(y^{1}, \ldots, y^{N-1}\right)$.

The coordinate $y^{N}$ refers to a distance along the inward normal to $C$ at the point $\left(y \ldots, y^{N-1}\right)$. The metric tensor has the form

$$
\begin{aligned}
& g_{\alpha \beta}=\left(G,,_{\alpha}^{k}+y^{N} X,_{\alpha}^{k}\right)\left(G,{ }_{\beta}^{k}+y^{N} X,{ }_{\beta}^{k}\right), \\
& g_{\alpha N}=\left(G,,_{\alpha}^{k}+y^{N} X,_{\alpha}^{k}\right) X^{k}=\frac{1}{2} y^{N}\left(X^{k} X^{k}\right),,_{\alpha}=0, \\
& g_{N N}=X^{k} X^{k}=1
\end{aligned}
$$

The Christoffel symbols which involve the normal coordinate $y^{N}$ are

$$
\begin{aligned}
& \left\{\begin{array}{c}
\rho \\
N \sigma
\end{array}\right\}=\frac{1}{2} g^{\rho \mu} \frac{\partial g_{\mu \sigma},}{\partial y^{N}} \\
& \left\{\begin{array}{c}
N \\
\rho \sigma
\end{array}\right\}=-\frac{1}{2} \frac{\partial g_{\rho \sigma}}{\partial y^{N}} \\
& \left\{\begin{array}{c}
N \\
N \rho
\end{array}\right\}=\left\{\begin{array}{c}
\rho \\
N, N
\end{array}\right\}=\left\{\begin{array}{c}
N \\
N N
\end{array}\right\}=0 .
\end{aligned}
$$

The various second order covariant derivatives of $u$ then have the form

$$
\begin{aligned}
& \bar{u}_{\mid \alpha \beta}=\frac{\partial^{2} \bar{u}}{\partial y^{\alpha} \partial y^{\beta}}-\left\{\begin{array}{c}
k \\
\alpha \beta
\end{array}\right\} \bar{u}_{\mid k}, \\
& \bar{u}_{\mid \alpha N}=\bar{u}_{\mid N \alpha}=\frac{\partial^{2} \bar{u}}{\partial y^{\alpha} \partial y^{N}}-\left\{\begin{array}{c}
\beta \\
\alpha N
\end{array}\right\} \bar{u}_{\mid \beta}, \\
& \bar{u}_{i N N}=\frac{\partial^{2} \bar{u}}{\left(\partial y^{N}\right)^{2}} .
\end{aligned}
$$

We indicate with a bar when the tensor is expressed in geodesic normal coordinates. Our operator takes the form

$$
L u=\left(a^{i j} u,{ }_{j}\right)_{\mid i}=\left(\bar{a}^{i j} \bar{u},{ }_{j}\right)_{\mid i} .
$$


When $L$ is the Laplace operator $\Delta$ we have

$$
\Delta u=\delta^{i j} u,{ }_{i j}=\bar{u},_{N N}+g^{\alpha \beta} \bar{u}_{\mid \alpha \beta},
$$

since $g^{\alpha N}=0$ and $g^{N N}=1$. Also the first invariant takes the form

$$
|\operatorname{grad} u|^{2}=\left(\bar{u},_{N}\right)^{2}+g^{\alpha \beta} \bar{u},{ }_{\alpha} \bar{u}, \beta .
$$

We now express the boundary integral appearing in (2.4) in geodesic normal coordinates.

$$
\begin{gathered}
-\oint_{C}\left\{\bar{f}^{N} \bar{a}^{i j}-2 \bar{f}^{j} \bar{a}^{i N}\right\} \bar{u},_{i} \bar{u},{ }_{j} d S=-\oint_{C}\left\{\overline { f } ^ { N } \left[\left(\overline{a^{\alpha \beta}} \bar{u},{ }_{\alpha} \bar{u}, \beta\right)\right.\right. \\
\left.-\bar{a}^{N N}\left(\bar{u},{ }_{N}\right)^{2}\right]-2\left(\bar{f} \bar{u}^{\alpha},{ }_{\alpha}\right)\left(\bar{u}^{i N} \bar{u},{ }_{i}\right\} d S .
\end{gathered}
$$

If $L \equiv \Delta$ then $\bar{a}^{i j}=g^{i j}$ and (2.17) becomes

$$
-\oint_{C}\left\{\bar{f}^{N}\left[\left(g^{\alpha \beta} \bar{u},{ }_{\alpha} \bar{u}, \beta\right)-\left(\bar{u},_{N}\right)^{2}\right]-2\left(\overline{f^{\alpha}} \bar{u},{ }_{\alpha}\right) \bar{u},_{N}\right\} d S .
$$

The surface integral in (2.6) can be treated in a similar manner to obtain

$$
\begin{aligned}
-\oint_{C} \bar{A}^{k l}\left\{\bar{f}^{N}\left[\bar{a}^{\alpha \beta} \bar{u}_{\mid k \alpha} \bar{u}_{\mid l \beta}-\bar{a}^{N N}\left(\bar{u}_{\mid k N}\right)\left(\bar{u}_{\mid l N}\right)\right]\right. \\
\left.-2\left(\bar{f}^{\alpha} \bar{u}_{\mid k \alpha}\right)\left(\bar{a}^{i N} \bar{u}_{\mid i l}\right)\right\} d S .
\end{aligned}
$$

In particular if $\bar{f}^{i}, \bar{A}^{k l}$ is chosen on $C$ so that

$$
\begin{gathered}
f^{N}=\bar{A}^{N N}=1, \\
f^{\alpha}=\bar{A}^{\alpha j}=0,
\end{gathered}
$$

then for $L \equiv \Delta(2.19)$ takes the simple form

$$
-\oint_{C}\left\{g^{\alpha \beta} \bar{u}_{\mid N \alpha} \bar{u}_{\mid N \beta}-\left(\bar{u}_{\mid N N}\right)^{2}\right\} d S .
$$

In view of (2.15) we have thereby isolated an interesting combination of mixed normal and tangential second derivatives on $C$ in terms of second order tangential derivatives on $C$ and certain integrals over $R$.

In fact, a specific mixed derivative, say $\bar{u}_{\mid N \sigma}$, can be isolated in this manner. Let

$$
\begin{aligned}
\bar{f}^{N} & =1, \quad \bar{f}^{\alpha}=0, \\
\bar{A}^{\sigma \sigma} & =1, \bar{A}^{i j}=0 \quad i, j \neq \sigma
\end{aligned}
$$

Then for $L \equiv \Delta(2.19)$ becomes

$$
-\oint_{C}\left\{g^{\alpha \beta} \bar{u}_{\mid \sigma \alpha} \bar{u}_{\mid \sigma \beta}-\left(\bar{u}_{\mid N \sigma}\right)^{2}\right\} d S .
$$

As we shall see in the next section, these choices of $\bar{f}^{i}, \bar{A}^{k l}$ will enable us to obtain new pointwise bounds for derivatives in certain boundary problems.
The following notation will be used throughout this paper. Let $f(x)$ be a piecewise continuous function on $R+C$, then

$$
\begin{aligned}
& f_{M}= \max f(x), \quad f_{m}= \\
& x \in R+C \min f(x) . \\
& x \in R+C
\end{aligned}
$$

For $N=2, x^{i}=G^{i}\left(y^{1}\right)$ represents a plane closed curve. Equation (2.10) becomes

$$
\chi^{i}=G^{i}(S)+N n^{i}(S)
$$

where we have set $y^{1}=S$ (arclength along $C$ ) and $y^{2}=N$ (distance along the unit inward normal $\left(n^{1}, n^{2}\right)$. If $K(S)$ is the curvature of $C$ then we have

$$
g^{11}=(1-K(S) N)^{-2}, \quad g^{12}=g^{21}=0, \quad g^{22}=1 .
$$

The use of this coordinate system is, of course, restricted to a certain strip in the neighborhood of the boundary where in terms of it all points are uniquely defined.

\section{Pointwise Bounds}

As was previously noted, Payne and Weinberger [6] made use of (2.4) in order to obtain bounds for solutions of second order elliptic partial differential equations. This identity enabled them to estimate the integral over a closed surface of the square of the normal derivative of a solution of a second order equation in terms of integrals of the squares of the function and its tangential derivative over the surface. In order to bound the derivatives at a point within the region they noted that one could differentiate the "Green's Identity," being careful to define a "parametrix" in such a way that this is permissible. The resulting estimates have the disadvantage that the coefficients become infinite more rapidly as the boundary is approached than do those in the estimates for the value of the solution itself. This procedure has also been used by Diaz [3] in estimating derivatives at a point for solutions of Laplace's equation.

One could proceed in an alternate manner. That is, instead of differentiating the "Green's Identity," simply write the identity using for the function to be evaluated at the point, the derivative of the solution. This leads to the estimation of surface integrals of squares of second derivatives of the function over the surface in terms of surface in tegrals of squares of tangential derivatives of the function over the surface. The identity (2.4) gives a means of obtaining the necessary inequalities.

As an example of an application of these identities we consider the problem of obtaining a priori pointwise bounds for the derivatives of a function $u$ for which $L u \equiv \Delta u-p u$ is known in $R+C$ and $u$ is known on $C$. Here $R$ is a simply connected finite plane region bounded by the smooth closed curve $C, \Delta$ is the Laplace operator and $p(x, y)>0$ and 
bounded together with its first derivatives in $R+C$. (The function $p$ is taken to be positive in $R+C$ for the sake of convenience. By a slight modification, similar results could be obtained for $p \geq 0$.) We assume that $L u$ and $u$ are sufficiently smooth in $R$ and on $C$ respectively.

As a starting point we use the "Green's Identity"

$$
(P)=\int_{R} \Gamma \Delta v d A+\oint_{C}\left(v \frac{\partial \Gamma}{\partial n}-\Gamma \frac{\partial v}{\partial n}\right) d S,
$$

where $\nu$ is any sufficiently smooth function in $R+C$, $P$ is a point in the in terior of $R, \Gamma=-\frac{1}{2 \pi} \ln r_{P Q}\left(r_{P Q}=\right.$ distance from the point $P$ to another point $Q)$, and $\frac{\partial}{\partial n}$ is the outward normal derivative. Now let $\nu=u,,_{i} \equiv \frac{\partial u}{\partial x_{i}}$. Then we have

$u,_{i}(P)=\int_{R} \Gamma \Delta u,{ }_{i} d A+\oint_{C}\left(u,,_{i} \frac{\partial \Gamma}{\partial n}-\Gamma \frac{\partial u_{, i}}{\partial n}\right) d S$,

which may be written

$$
\begin{array}{r}
\chi_{i}(P) \equiv u,{ }_{i}(P)-\int_{R} \Gamma(\Delta u-p u),_{i} d A=\int_{R} \Gamma(p u),_{i} \\
+\oint_{C}\left(u,,_{i} \frac{\partial \Gamma}{\partial n}-\Gamma \frac{\partial u,,_{i}}{\partial n}\right) d S .
\end{array}
$$

We shall consider $\chi_{i}(P)$, since $\chi_{i}(P)--u,{ }_{i}(P)$ is assumed known. Making use of Schwarz's inequality for vectors it follows that

$$
\begin{aligned}
\chi_{i}(P) \chi_{i}(P) \leq & K_{\Gamma}\left(\frac{1}{2} \int_{R} p u^{2} d A+D(u, u)\right. \\
& \left.+\oint_{C} u,{ }_{i} u,{ }_{i} d S+\oint_{C} \frac{\partial u_{\cdot i}}{\partial n} \frac{\partial u_{\cdot i}}{\partial n} d S\right)
\end{aligned}
$$

where $D(u, u)$ is Dirichlet integral, and

$$
\begin{aligned}
K_{\Gamma}=8 \int_{R} \Gamma^{2}|\operatorname{grad} \sqrt{p}|^{2} d A & +\int_{R} \Gamma^{2} p^{2} d A \\
& +\oint_{C}\left(\frac{\partial \Gamma}{\partial n}\right)^{2} d S+\oint_{C} \Gamma^{2} d S .
\end{aligned}
$$

Now we have

$$
\oint_{C} \frac{\partial u_{i}}{\partial n} \frac{\partial u, i_{i}}{\partial n} d S \leq \oint_{C} u,,_{i j} u,,_{j} d S
$$

and in terms of the normal coordinate system introduced in section 2 (with the boundary coordinate taken to be arc length, i.e., $y^{1}=S, y^{2}=N$ ),

$$
\oint_{C} u_{, i j} u,{ }_{i j} d S=\oint_{C} u_{\mid N N}^{2}+2 u_{\mid N S}^{2}+u_{\mid S S}^{2} d S .
$$

Now on $C, \Delta u-p u=u_{\mid N N}+u_{\mid S S}-p u$, so that

$$
\begin{aligned}
& \oint_{C} u,{ }_{i j} u,{ }_{i j} d S=\oint_{C}\left[p u-u_{\mid S, S}-(\Delta u-p u)\right]^{2} \\
& +u_{T_{S S}}^{2} d S+2 \oint_{C} u_{\uparrow_{N S}}^{2} d S .
\end{aligned}
$$

Noting that

$$
u_{\mid S S}=\frac{\partial^{2} u}{\partial s^{2}}-K \underset{\partial N}{\partial u}=\frac{\partial^{2} u}{\partial s^{2}}+K \frac{\partial u}{\partial n}
$$

we have that

$$
\begin{aligned}
\oint_{C} u_{i, j} u_{, i j} d S & \leq 2 \oint_{C}\left[\left(p u-\frac{\partial^{2} u}{\partial s^{2}}-[\Delta u-p u\rfloor\right)^{2}+\left(\frac{\partial u}{\partial S^{2}}\right)^{2}\right] d S \\
& +4 K_{M}^{2} \oint_{C}\left(\frac{\partial u}{\partial n}\right)^{2} d S+2 \oint_{C} u_{\mid N S}^{2} d S .
\end{aligned}
$$

It follows from Green's Identity and the arithmeticgeometric mean inequality

$$
2 \alpha a \frac{b}{\alpha} \leq \alpha^{2} a^{2}+\frac{b^{2}}{\alpha^{2}}, \quad \alpha>0
$$

that

$\frac{1}{2} \int_{R} p u^{2} d A+D(u, u) \leq \oint_{C} u \frac{\partial u}{\partial n} d S$

$$
+\frac{1}{2} \int_{R} \frac{(\Delta u-p u)^{2}}{p} d A .
$$

Combining (3.10), (3.12) with (3.4) we obtain

$$
\begin{aligned}
\chi_{i}(p) \chi_{i}(p) \leq & K_{\Gamma}\left(\frac{1}{2} \int_{R} \frac{(\Delta u-p u)^{2}}{p} d A\right. \\
+2 \oint_{C} & {\left[\left(p u-\frac{\partial^{2} u}{\partial S^{2}}\right)^{2}+\left(\frac{\partial^{2} u}{\partial S^{2}}\right)^{2}\right] d S+\oint_{C}\left(\frac{\partial u}{\partial S}\right)^{2} d S } \\
& \left.+\frac{1}{2} \oint_{C} u^{2} d S+\frac{11}{2} \oint_{C}\left(\frac{\partial u}{\partial n}\right)^{2} d S+2 \oint_{C} u_{N_{N S}} d S\right) .
\end{aligned}
$$

A.ll terms on the right hand side of (3.13) except the last two are in terms of data. In order to bound the integral of the square of the normal derivative around the boundary we write the identity

$$
\begin{aligned}
\oint_{C}\left[f^{k} n_{k}\right. & \left.\left(\left(\frac{\partial u}{\partial s}\right)^{2}-\left(\frac{\partial u}{\partial n}\right)^{2}\right)-2 f^{i} s_{i} \frac{\partial u}{\partial n} \frac{\partial u}{\partial s}\right] d S \\
= & \int_{R}\left[f^{k}{ }_{, k} \delta^{i j}-f_{, j}^{i}-f_{, i}^{j}\right] u,{ }_{,} u,{ }_{j} d A-2 \int_{R} f^{i} u,{ }_{i} \Delta u d A
\end{aligned}
$$

which has its left hand side essentially in the form of (2.18). Here $a^{i j}=\delta^{i j}, n_{i}$ and $s_{i}$ are the unit normal and unit tangent vectors respectively and $f^{i}$ is 
an arbitrary continuous, piecewise continuously differentiable vector field in $R+C$. We choose $f^{k}$ such that $f^{k} n_{k}>0$ on $C$. (For example, if $R$ is star-shaped with respect to the origin, then we may take $f^{k}=x^{k}$.) From (3.14) we obtain the inequality

$$
\begin{aligned}
& \frac{3}{4}\left(f^{k} n_{k}\right)_{m} \oint_{C}\left(\frac{\partial u}{\partial n}\right)^{2} d S \\
& \leq \oint_{C}\left[\left(f^{k} n_{k}\right)+\frac{4\left(f^{k} s_{k}\right)^{2}}{\left(f^{k} n_{k}\right)}\right]\left(\frac{\partial u}{\partial s}\right)^{2} d S \\
& +\left(C_{1}+K_{1}+K_{2}\right) D(u, u)+\frac{\left(f^{i} f^{i} p\right)_{M}}{K_{1}} \int_{R} p u^{2} d A \\
& +\frac{1}{K_{2}} \int_{R} f^{i} f^{i}(\Delta u-p u)^{2} d A
\end{aligned}
$$

where $C_{1}$ is any bound for the largest eigenvalue of the coefficient matrix $\left[-f_{, k}^{k} \delta^{i j}+f_{, j}^{i}+f_{, i}^{i}\right]$, and $K_{1}$ and $K_{2}$ are positive. Let $c=\max \left[C_{1},\left(f^{i} f^{i} p\right)_{M}\right]$ and choose $c+K_{1}+K_{2}=\frac{2 c}{k_{1}}$. (For example, take $K_{1}=\frac{-c+\sqrt{c^{2}+4 c}}{2}$ and $K_{2}=\frac{c}{K_{1}}$.) Then we have, using (3.12) and (3.11) with (3.15),

$$
\begin{aligned}
& \frac{1}{2}\left(f^{k} n_{k}\right)_{m} \oint_{C}\left(\frac{\partial u}{\partial n}\right)^{2} d S \leq \oint_{C}\left[f^{k} n_{k}\right. \\
& \left.+\frac{4\left(f^{j} s_{j}\right)^{2}}{\left(f^{i} n_{i}\right)}\right]\left(\frac{\partial u}{\partial S}\right)^{2} d S+\frac{K_{1}}{c} \int_{R} f^{i} f^{i}(\Delta u-p u)^{2} d A \\
& +\frac{c}{K_{1}} \int_{R} \frac{(\Delta u-p u)^{2}}{p} d A+\frac{4 c^{2}}{K_{1}^{2}} \oint_{C} \frac{u^{2}}{\left(f^{i} n_{i}\right)} d S
\end{aligned}
$$

or for simplicity

$$
\begin{aligned}
\oint_{C}\left(\frac{\partial u}{\partial n}\right)^{2} d S \leq a_{1} \oint_{C} u^{2} d S & +a_{2} \oint_{C}\left(\frac{\partial u}{\partial s}\right)^{2} d S \\
& +a_{3} \oint_{C}(\Delta u-p u)^{2} d S
\end{aligned}
$$

where

$$
\begin{aligned}
& a_{1}=\frac{8 c^{2}}{K^{2}\left(f^{k} n_{k}\right)_{m}^{2}} \\
& a_{2}=2\left(f^{k} n_{k}\right)_{m}^{-1}\left[f^{k} n_{k}+\frac{f^{j} s_{j}}{\left(f^{j} n_{j}\right)_{m}}\right]_{M} \\
& a_{3}=\frac{2}{\left(f^{k} n_{k}\right)_{m}}\left\{\frac{K_{1}}{c}\left(f^{i} f^{i}\right)_{M}+\frac{c}{K_{1}} \frac{1}{p_{m}}\right\}
\end{aligned}
$$

Finally, we need to obtain a bound for $\oint_{C} u_{\mid N S}{ }_{\mid N S}$. We make use of (2.23) and (2.6) assuming that the vector field $f^{i}$ and the tensor field $A^{i j}$ have been chosen as indicated. We then obtain the identity

$$
\begin{gathered}
\oint_{C}\left(u_{\mid N S}^{2}-u_{\mid, S S}^{2}\right) d S=-2 \int_{R} f^{m} A^{i j} u_{\mid j m}(\Delta u)_{\mid i} d A \\
\quad+\int_{R}\left\{\left(f^{m} A^{i j}\right)_{\mid m} g^{k l}-2\left(f^{l} A^{i j}\right)_{\mid k}\right\} u_{\mid i k} u_{\mid j l} d A .
\end{gathered}
$$

We assume also that $f^{i}$ and $A^{i j}$ are bounded together with their derivatives in $R+C$. (If the boundary is sufficiently smooth one can define these tensors (in the $(S, N)$ system) as follows. Let

$$
\begin{aligned}
& f^{1}=0, \quad f^{2}=(1-\bar{K} N) \\
& \left.A^{22}=A^{12}=A^{21}=0, \quad A^{11}=(1-\bar{K} N)\right\} \text { for } N \leq \frac{1}{\bar{K}} \\
& f^{i} \equiv 0, \quad A^{i j} \equiv 0 \\
& \text { for } N>\frac{1}{\bar{K}}
\end{aligned}
$$

where $\bar{K}$ is a constant greater than the maximum curvature $K_{M}$ of $C$.

Using (3.11) and Schwarz's inequality we obtain from (3.18)

$$
\begin{aligned}
\oint_{C} u_{\mid N S S}^{2} d S & \leq \oint_{C} u_{\mid S S}^{2} d S+\int_{R}(\Delta u-p u),{ }_{i}(\Delta u-p u),{ }_{i} d A \\
& +\int_{R}(p u),{ }_{i}(p u){ }_{i} d A+\int_{R} C^{i j k l} u_{\mid i k} u_{\mid j l} d A
\end{aligned}
$$

where

$C^{i j k l}=2 f^{k} f^{l} A^{m i} A^{n j} g_{m n}+\left(f^{m} A^{i j}\right)_{\mid m} g^{k l}-2\left(f^{l} A^{i j}\right)_{\mid k}$.

The last term on the right hand side of (3.19) is bounded as follows:

$$
\int_{R} C^{i j k l} u_{\mid i k} u_{|j\rangle} d A \leq B \int_{R} u,{ }_{i j} u,,_{i j} d A
$$

where $B$ is a constant. After a moderate calculation, making use of the definitions of $f^{i}$ and $A^{i j}$ in the normal coordinate system, it is possible to obtain the bound

$$
B=1+\bar{K}-K_{m}+\max \left(\overline{K,} 2\left|2\left(K^{\prime}\right)^{2} / \overline{K^{3}}-K\right|_{M}\right)
$$

where $K^{\prime}$ is the derivative of the curvature with respect to are length. Now using the divergence theorem we have

$$
\begin{aligned}
\int_{R} u,{ }_{i j} u,{ }_{i j} d A=\oint_{C} u,,_{i}\left(n_{j} \frac{\partial}{\partial x_{i}}-n_{i}\right. & \left.\frac{\partial}{\partial x_{j}}\right) u, d S \\
& +\int_{R}(\Delta u)^{2} d A
\end{aligned}
$$

where the $n_{i}$ are Cartesian components of the exterior unit normal. We may rewrite the boundary integral in terms of normal and tangential derivatives and obtain

$$
\begin{aligned}
& \int_{R} u,{ }_{, i j} u,{ }_{, j} d A=+2 \oint_{C} \frac{\partial u}{\partial n} \frac{\partial^{2} u}{\partial s^{2}} d S \\
& +\oint_{C} K\left(\left(\frac{\partial u}{\partial n}\right)^{2}+\left(\frac{\partial u}{\partial s}\right)^{2}\right) d S+\int_{R}(\Delta u)^{2} d A .
\end{aligned}
$$


Using (3.11) we have

$$
\begin{aligned}
\int_{R} u,{ }_{i j} u,{ }_{i j} d A \leq \oint_{C} K\left(\frac{\partial u}{\partial S}\right)^{2} d S & +\oint_{C}\left(\frac{\partial^{2} u}{\partial S^{2}}\right)^{2} d S \\
+\oint_{C}(1+K)\left(\frac{\partial u}{\partial n}\right)^{2} d S & +2 \int_{R}(\Delta u-p u)^{2} d A \\
& +2 \int_{R}(p u)^{2} d A .
\end{aligned}
$$

Using (3.11) and (3.12) we have the inequality

$$
\begin{aligned}
& \int_{R}(p u)^{2} d A \leq p_{M}\left\{\oint_{C} u^{2} d S\right. \\
&\left.+\oint_{C}\left(\frac{\partial u}{\partial n}\right)^{2} d S+\int_{R} \frac{(\Delta u-p u)^{2}}{p} d A\right\} .
\end{aligned}
$$

Combining (3.17), (3.24) and (3.25) we have

$$
\begin{aligned}
\int_{R} u,{ }_{i j} u,{ }_{i j} d A \leq b_{1} \oint_{C} u^{2} d S+b_{2} \oint_{C}\left(\frac{\partial u}{\partial S}\right)^{2} d S & \\
& +b_{3} \oint_{C}\left(\frac{\partial^{2} u}{\partial S^{2}}\right)^{2} d S+b_{4} \int_{R}(\Delta u-p u)^{2} d A
\end{aligned}
$$

where

$b_{1}=\left[(1+K)_{M}+2 p_{M}\right] a_{1}+2 p_{M}, \quad b_{2}=\left[(1+K)_{M}+2 p_{M}\right) a_{2}$

$+K_{M}, b_{3}=1$ and $b_{4}=2+\left[(1+K)_{M}+2 p_{M}\right] a_{3}+2 p_{M} / p_{m}$

Now in (3.19) we are left to consider the terms

$$
\oint_{C} u_{\uparrow_{S S S}} d S \text { and } \int_{R}(p u),{ }_{i}(p u),{ }_{i} d A .
$$

In order to bound the latter we can write

$$
\begin{aligned}
& \int_{R}(p u),{ }_{i}(p u),{ }_{i} d A \\
& =\int_{R}\left(p,{ }_{i} p,{ }_{i} u^{2}+2 p u,{ }_{i} p,{ }_{i} u+p^{2} u,{ }_{i} u,{ }_{i}\right) d A \\
& \leq \int_{R}\left(\frac{2 p,{ }_{i} p,{ }_{i}}{p}+p^{2}\right)\left(\frac{1}{2} p u^{2}+u,{ }_{i} u,{ }_{i}\right) d A
\end{aligned}
$$

where (3.11) has been used with $\alpha=\sqrt{\frac{2 p,,_{i} p,_{i}}{p}}$.

Now (3.27) and (3.12) yield

$$
\begin{aligned}
\int_{R}(p u),_{i}(p u),_{i} d A & \\
\leq \frac{1}{2}\left(\frac{2 p,,_{i} p,,_{i}}{p}+p^{2}\right){ }_{M} & \left\{\oint_{C} u^{2} d S+\oint_{C}\left(\frac{\partial u}{\partial n}\right)^{2} d S\right. \\
& \left.+\int_{R} \frac{(\Delta u-p u)^{2}}{p} d A\right\}
\end{aligned}
$$

and hence, using (3.17) we have

$$
\begin{aligned}
\int_{R}(p u){ }_{i}(p u),{ }_{i} d A \leq c_{1} \oint_{C} u^{2} d S & \\
& +c_{2} \oint_{C}\left(\frac{\partial u}{\partial s}\right)^{2} d S+c_{3} \oint_{C}(\Delta u-p u)^{2} d S
\end{aligned}
$$

where

$$
\begin{aligned}
c_{1} & =\frac{1}{2}\left(\frac{2 p,{ }_{i} p,,_{i}}{p}+p^{2}\right)_{M}\left(1+a_{1}\right), \\
c_{2} & =\frac{1}{2}\left(\frac{2 p,{ }_{i} p,,_{i}}{p}+p^{2}\right)_{M} a_{2}
\end{aligned}
$$

and

$$
c_{3}=\frac{1}{2}\left(\frac{2 p,{ }_{i} p,_{i}}{p}+p^{2}\right)_{M}\left(a_{3}+\frac{1}{p_{M}}\right) .
$$

Finally we have from (3.9), (3.11), and (3.17)

$$
\begin{array}{r}
\oint_{C} u_{\mid S S}^{2} d S \leq 2 K_{M}^{2} a_{1} \oint_{C} u^{2} d S+2 K_{M}^{2} a_{2} \oint\left(\frac{\partial u}{\partial s}\right)^{2} d S \\
+2 \oint_{C}\left(\frac{\partial^{2} u}{\partial S^{2}}\right)^{2} d S+2 K_{M}^{2} a_{3} \int_{R}(\Delta u-p u)^{2} d A .
\end{array}
$$

Combining (3.19), (3.21), (3.26), (3.29) and (3.30) we have

$$
\begin{gathered}
\oint_{C} u_{\mid N S}^{2} d S \leq d_{1} \oint_{C} u^{2} d S+d_{2} \oint_{C}\left(\frac{\partial u}{\partial S}\right)^{2} d S+d_{3} \oint_{C}\left(\frac{\partial^{2} u}{\partial S^{2}}\right)^{2} d S \\
\quad+d_{4} \int_{R}(\Delta u-p u)^{2} d A+d_{5} \int_{R}(\Delta u-p u),{ }_{i}(\Delta u-p u),{ }_{i} d A
\end{gathered}
$$

where

$$
\begin{aligned}
& d_{1}=2 K_{M}^{2} a_{1}+B b_{1}+c_{1}, d_{2}=2 K_{M}^{2} a_{2}+B b_{2}+c_{2}, \\
& d_{3}=2+B b_{3}, d_{4}=2 K_{M}^{2} a_{3}+B b_{4}+c_{3} \text { and } d_{5}=1 .
\end{aligned}
$$

Now (3.17) and (3.31) may be inserted into (3.13) to yield the desired bound,

$$
\begin{aligned}
\chi_{i} \chi_{i}(P) \leq & K_{\Gamma}\left\{\beta_{1} \oint_{C} u^{2} d S+\beta_{2} \oint_{C}\left(\frac{\partial u}{\partial S}\right)^{2} d S\right. \\
+ & \beta_{3} \oint_{C}\left(\frac{\partial^{2} u}{\partial s^{2}}\right)^{2} d S+\beta_{5} \int_{R}(\Delta u-p u)^{2} d A \\
& \left.+\beta_{4} \oint_{R}(\Delta u-p u)_{, i}(\Delta u-p u)_{,} d A\right\}
\end{aligned}
$$

where the $\beta_{j}$ 's are explicitly determined constants.

It should be pointed out that for the sake of simplicity, no effort was made to obtain the "best" such bound. Better bounds could be obtained, for example, by leaving certain known quantities under the sign of integration (compare equations (3.16) and (3.17)) rather than replacing them by a maximum value. 


\section{Higher Derivatives in N Dimensions}

The process described in section 3 can be generalized easily to higher derivatives. In bounding the $M$ th derivative we must require the existence and integrability of $M$ th derivatives of $\Delta u-p u, p u$, and $M+1$ tangential derivatives of $u$ on $C$. We also assume that bounds have been obtained for the various lower order differential invariants.

The mode of generalization will become apparent from the case $M=2$ where we assume that bounds have been obtained for

$$
\int_{R} p u^{2} d v, D(u, u), \int_{R} u,{ }_{i j} u,,_{j} d v, \oint_{C} \frac{\partial u}{\partial n} d S, \oint_{C} u,{ }_{i j} u,{ }_{i j} d S
$$

as was done explicitly in two dimensions in the previous section.

If $\omega_{n}$ denotes the surface area of the unit sphere in $N$ dimensions then

$$
\Gamma=\frac{-1}{(N-2) \omega_{n} r_{P Q}^{N-2}}, N>2
$$

is a fundamental solution of $\Delta u=0$. We can write

$$
\begin{aligned}
\chi_{i j}(P) \equiv u,{ }_{i j}(P)-\int_{R} \Gamma(\Delta u-p u),{ }_{i j} d v=\int_{R} \Gamma(p u),{ }_{i j} d v \\
+\oint_{C}\left(u,{ }_{i j} \frac{\partial \Gamma}{\partial n}-\Gamma \frac{\partial u,,_{i j}}{\partial n}\right) d S
\end{aligned}
$$

Since $\int_{R} \Gamma^{2} d v$ does not exist for $N \geq 4$ we cannot apply Schwarz's inequality, as was done in (3.4), for dimensions higher than three.

For $N \geq 4$ we apply Schwarz's inequality as follows

$$
\left(\int_{R} \Gamma u d v\right)^{2} \leq \int_{R} r^{N-1} \Gamma^{2} d v \int_{R} r^{-(N-1)} u^{2} d v
$$

where the singularity of $\Gamma$ is chosen as the origin. In order to bound $\int_{R} r^{-(N-1)} u^{2} d v$ we use "Green's Identity"

$-\int_{R}\left[\Delta\left(r^{-(N-3)}\right) u^{2}-r^{-(N-3)} \Delta u^{2}\right] d \nu$

$=$ Boundary Integrals.

Now using (3.11) we can easily obtain

$$
\begin{aligned}
\int_{R} r^{-(N-1)} u^{2} d v \leq \frac{4}{2 N-7} \int_{R} r^{-(N-3)}(\Delta u-p u)^{2} d v & \\
& + \text { Boundary Integrals. }
\end{aligned}
$$

This same basic technique may be used to handle all terms in (4.3) for $N \geq 4$.
The boundary integrals occurring in the above inequalities are the same as those to be dealt with below.

We shall show how the desired bounds may be obtained for $N \leq 3$ since in higher dimensions only technical details enter.

Applying Schwarz's inequality, as was done in (3.4), we see that

$$
\begin{aligned}
\chi_{i j}(P) \chi_{i j}(P) & \leq K_{\Gamma}\left\{\int_{R}(p u)_{, i j}(p u)_{, i j} d v\right. \\
& \left.+\oint_{C} u,_{i j} u,,_{i j} d S+\oint_{C} \frac{\partial u,_{i j}}{\partial n} \frac{\partial u,{ }_{i j}}{\partial n} d S\right\}
\end{aligned}
$$

where $K_{\Gamma}$ is given by (3.5).

We see that

$$
\begin{aligned}
& \int_{R}(p u)_{, i j}(p u)_{, i j} d v \leq 3\left(\frac{p,{ }_{i j} p,,_{i j}}{p}\right)_{M} \int_{R} p u^{2} \\
& +12\left(p,,_{i} p,{ }_{i}\right)_{M} D(u, u)+3 p_{M}^{2} \int_{R} u,{ }_{i j} u,{ }_{i j} d v
\end{aligned}
$$

but by assumption each integral on the right side of (4.5) has a known bound. Similarly we assume that a bound is known for $\oint_{C} u, i_{i j} u,{ }_{i j} d S$.

It now remains for us to obtain bounds for the last term of (4.4). We see that

$\oint_{C} \frac{\partial u,_{i j}}{\partial n} \frac{\partial u,_{i j}}{\partial n} d s=\oint_{r} g^{i j} g^{k l} \bar{u}_{\mid i k N} \bar{u}_{\mid j l N} d S$

$$
\begin{aligned}
=\oint_{C} g^{\alpha \beta} g^{\gamma \delta} \bar{u}_{\mid \alpha \gamma N} \bar{u}_{\beta \delta \mid N} d S+ & 2 \oint_{C} g^{\alpha \beta} \bar{u}_{\mid \alpha N N} \bar{u}_{\mid B N N} d S \\
& +\oint_{C}\left(\bar{u}_{\mid N N N}\right)^{2} d S .
\end{aligned}
$$

From (2.15) we see that

$$
\bar{u}_{\mid \sigma N N}=(\Delta \bar{u}-\bar{p} \bar{u})_{\mid \sigma}+(\bar{p} \bar{u})_{\mid \sigma}-g^{\alpha \beta} \bar{u}_{\mid \alpha \beta \sigma} .
$$

The first two expressions on the right are known and

$$
\begin{aligned}
\bar{u}_{\mid \alpha \beta \gamma}=\frac{\partial}{\partial y^{\gamma}}\left[\frac{\partial^{2} \bar{u}}{\partial y^{\alpha} \partial y^{\beta}}-\right. & \left.\left\{\begin{array}{c}
k \\
\alpha \beta
\end{array}\right\} \bar{u}_{\mid k}\right] \\
& -\left\{\begin{array}{c}
k \\
\alpha \gamma
\end{array}\right\} \bar{u}_{\mid k \beta}-\left\{\begin{array}{c}
k \\
\beta \gamma
\end{array}\right\} \bar{u}_{\mid \alpha k},
\end{aligned}
$$

where

$$
\frac{\partial \bar{u}_{\mid k}}{\partial y^{\gamma}}=\bar{u}_{\mid \gamma k}+\left\{\begin{array}{c}
j \\
\gamma k
\end{array}\right\} \bar{u}_{\mid j} .
$$

The first term on the right side of (4.8) is known. We can use the inequalities

$$
\begin{aligned}
& b^{i j} \bar{u}_{\mid i} \bar{u}_{\mid j} \leq \tau_{1} g^{i j} \bar{u}_{\mid i} \bar{u}_{\mid j}, \\
& b^{i j k l} \bar{u}_{\mid i j} \bar{u}_{\mid k l} \leq \tau_{2} g^{i j} g^{k l} \bar{u}_{\mid i k} \bar{u}_{\mid j l},
\end{aligned}
$$


where $\tau_{1}, \tau_{2}$ are upper bounds for the largest eigenvalue in each case, to complete the bound for

$$
\oint_{C} g^{\alpha \beta} \bar{u}_{\mid \alpha N N} \bar{u}_{\mid \beta N N} d S
$$

by a function of the quantities (4.1).

Furthermore, from (2.15) we see that

$$
\bar{u}_{\mid N N N}=(\Delta \bar{u}-\bar{p} \bar{u})_{\mid N}+(\bar{p} \bar{u})_{\mid N}-g^{\alpha \beta} \bar{u}_{\mid \alpha \beta N}
$$

and hence we can bound the last term of (4.6) in terms of known quantities and a bound for

$$
\oint_{C} g^{\alpha \beta} g^{\gamma \delta} \bar{u}_{\mid \alpha \gamma N} \bar{u}_{\mid \beta \delta N} d S
$$

From (2.7) for $M=2$ and the choice

$$
\begin{aligned}
& \bar{f}^{N}=1, \quad \bar{f}^{\alpha}=0, \\
& \bar{A}^{\alpha \beta}=g^{\alpha \beta}, \quad \bar{A}^{i \alpha}=\bar{A}^{N N}=0 \\
& \bar{B}^{N N}=1, \quad \bar{B}^{i j}=0 \quad i, j \neq N,
\end{aligned}
$$

we have

$$
\begin{aligned}
\oint_{C}\left[g^{\alpha \beta} g^{\gamma \delta} \bar{u}_{\mid \alpha \gamma N} \bar{u}_{\mid \beta \delta N}-g^{\alpha \beta} \bar{u}_{\mid \alpha N N} \bar{u}_{\mid \beta N N}\right] d S \\
\quad=-\int_{R} f^{m} A^{i j} B^{k \imath}[(\Delta u-p u)+p u]_{\mid i k} u_{\mid j l m} d v \\
\quad+\int_{R}\left\{\left(f^{m} A^{i j} B^{k l} \delta^{r s}\right)_{\mid m}-2\left(f^{s} A^{i j} B^{k l}\right)_{\mid r}\right\} u_{\mid i k r} u_{\mid j l s} d V .
\end{aligned}
$$

\section{Hence}

$$
\begin{array}{r}
\oint_{C} g^{\alpha \beta} g^{\gamma \delta} \bar{u}_{\mid \alpha \gamma N} \bar{u}_{\mid \beta \delta N} d S \leq \oint_{C} g^{\alpha \beta} \bar{u}_{\mid \alpha N N} \bar{u}_{\mid \beta N N} d S \\
+\int_{R} A^{i j} B^{k l}\left[(\Delta u-p u)_{\mid i k}(\Delta u-p u)_{\mid j l}\right. \\
\left.\quad+(p u)_{\mid i k}(p u)_{\mid j l]}\right] d v+I\left(u_{\mid i j k}\right),
\end{array}
$$

where

$$
\begin{array}{r}
I\left(u_{i j \mid j k}\right)=\int_{R}\left\{\left(f^{m} A^{i j} B^{k l} \delta^{r s}\right)_{\mid m}-2\left(f^{s} A^{i j} B^{k l}\right)_{\mid r}\right. \\
\left.+f^{r} f^{s} A^{i j} B^{k l}\right\} u_{\mid i k r} u_{\mid j l s} d v .
\end{array}
$$

But

$$
\begin{aligned}
I\left(u_{\mid i j k}\right) \leq \tau_{3} \int_{R} u_{, i j k} u_{, i j k} d v & \\
= & \tau_{3}\left\{\oint_{C} u_{, i j} u_{, i j k} \nu_{k} d s-\int_{R}(\Delta u)_{, i j} u_{, i j} d v\right\} \\
= & \frac{\left(\tau_{3}\right)^{2}}{4 \epsilon} \oint_{C} g^{i j} g^{k l} \bar{u}_{\mid i k} \bar{u}_{\mid j l} d s-\tau_{3} \int_{R}(\Delta u)_{, i j} u_{, i j} d v \\
& +\epsilon \oint_{C} g^{i j} g^{k l} \bar{u}_{\mid i k N} \bar{u}_{\mid j l N} d s
\end{aligned}
$$

where $\tau_{3}$ is again an upper bound for the largest eigenvalue of the coefficient matrix in (4.15). We can substitute (4.1) into (4.4) for $\epsilon<1$ and achieve the desired bound for

$$
\oint_{C} g^{\alpha \beta} g^{\gamma \delta} \bar{u}_{\mid \alpha \gamma N} \bar{u}_{\mid \beta \delta N} d s .
$$

\section{References}

[1] S. Bergman and M. Schiffer, Kernel functions and elliptic differential equations in mathematical physics, Academic Press, New York (1953).

[2] J. H. Bramble and L. E. Payne, Bounds in the first biharmonic boundary value problem (to be published).

[3] J. B. Diaz, Upper and lower bounds for quadratic integrals, and at a point, for solutions of linear boundary value problems, Proc. Symp. Boundary Val. Probs. Differential Equations, U.S. Army Research Center, Univ. of Wisconsin, Apr. 20-22, 1959.

[4] L. Hörmander, Uniqueness theorems and estimates for normally hyperbolic partial differential equations of the second order, Compt. Rend. Douzième Congr. Mathématiciens Scandinaves Tenu à Lund, pp. 105-115 (1953).

[5] B. E. Hubbard, Bounds for eigenvalues of free and fixed membrane by finite difference methods, Pac. J. Math. 11, 559-590 (1961).

[6] L. E. Payne and H. F. Weinberger, New bounds for solutions of second order elliptic partial differential equations, Pac. J. Math. 8, No. 3, 551-573 (1958).

[7] F. Rellich, Darstellung der Eigenwerte von $\Delta u+\lambda u$ durch ein Randintegral, Math. Z. 46, 635-636 (1940).

[8] J. L. Synge and A. Schild, Tensor calculus (Univ. of Toronto Press, Toronto, 1952).

(Paper 65B4-65) 